\title{
Prompt photon and associated heavy quark production in the $\mathbf{k}_{\mathrm{T}}$-factorization approach
}

\author{
A.V. Lipatov, M.A. Malyshev* and N.P. Zotov \\ Skobeltsyn Institute of Nuclear Physics, Lomonosov Moscow State University \\ Moscow, Russia \\ E-mail: malyshevetheory.sinp.msu.ru
}

We present the results of the numerical calculations of prompt photon and associated heavy quark production at Tevatron and LHC in the framework of the $k_{T}$-factorization approach with KMR parton distributions. Our predictions are compared with the $\mathrm{D} \emptyset$ and $\mathrm{CDF}$ experimental data. Also we make predictions for LHC

XXI International Baldin Seminar on High Energy Physics Problems,

September 10-15, 2012

JINR, Dubna, Russia

*Speaker. 
Prompt photon and associated heavy $(b, c)$ quark production has been a subject of pointed theoretical and experimental investigations up to now since it is highly sensitive to parton distribution in the hadron. So it provides a test of hard subprocess dynamics. Also this process contributes to the background for the physics beyound the Standard Model processes.

In this work the $k_{T}$-factorization approach is used to make the calculations. It was used to describe the production of prompt photons associated with the charm or beauty quark in paper [1]. The consideration was based on the $\mathscr{O}\left(\alpha \alpha_{s}^{2}\right)$ amplitude for the gluon fusion subprocess $g^{*} g^{*} \rightarrow$ $\gamma Q \bar{Q}$. A reasonably good agreement between the numerical predictions and the Tevatron data $[2,3]$ was obtained in the region of relatively low $p_{T}^{\gamma}$ where the off-shell gluon fusion dominates. However, the quark-induced subprocesses become more important at moderate and large $p^{\gamma}$ and therefore should be taken into account. Here we extend the previous analysis by including into the consideration two additional $\mathscr{O}\left(\alpha \alpha_{s}^{2}\right)$ subprocesses: $q \bar{q} \rightarrow \gamma Q \bar{Q}$ and $q Q \rightarrow \gamma q Q$, where $Q$ is the charm or beauty quark.

The essential ingredient of the $k_{T}$-factorization approach is unintegrated (transeverse momentum dependent) parton distribution functions (uPDFs). One of the aims of the presented study was to test these parton densities. This is important as a step in the search of universal universal uPDFs.

According to the $k_{T}$-factorization theorem, to calculate the cross section of the prompt photon and associated heavy quark production one should convolute the off-shell partonic cross sections with the relevant unintegrated quark and/or gluon distributions in a proton:

$$
\sigma=\sum_{a, b=q, g} \int \hat{\sigma}_{a b}^{*}\left(x_{1}, x_{2}, \mathbf{k}_{1 T}^{2}, \mathbf{k}_{2 T}^{2}\right) f_{a}\left(x_{1}, \mathbf{k}_{1 T}^{2}, \mu^{2}\right) f_{b}\left(x_{2}, \mathbf{k}_{2 T}^{2}, \mu^{2}\right) d x_{1} d x_{2} d \mathbf{k}_{1 T}^{2} d \mathbf{k}_{2 T}^{2} \frac{d \phi_{1}}{2 \pi} \frac{d \phi_{2}}{2 \pi},
$$

where $\hat{\sigma}_{a b}^{*}\left(x_{1}, x_{2}, \mathbf{k}_{1 T}^{2}, \mathbf{k}_{2 T}^{2}\right)$ is the relevant partonic cross section. The initial off-shell partons have fractions $x_{1}$ and $x_{2}$ of initial protons longitudinal momenta, non-zero transverse momenta $\mathbf{k}_{1 T}$ and $\mathbf{k}_{2 T}$ and azimuthal angles $\phi_{1}$ and $\phi_{2}$.

In this work we test the KMR uPDFs [4, 5]. The KMR approach is the formalism to construct the unintegrated parton distributions $f_{a}\left(x, \mathbf{k}_{T}^{2}, \mu^{2}\right)$ from the known conventional parton distributions $x a\left(x, \mu^{2}\right)$, where $a=g$ or $a=q$. In this approximation, the unintegrated quark and gluon distributions are given by $[4,5]$

$$
\begin{gathered}
f_{q}\left(x, \mathbf{k}_{T}^{2}, \mu^{2}\right)=T_{q}\left(\mathbf{k}_{T}^{2}, \mu^{2}\right) \frac{\alpha_{s}\left(\mathbf{k}_{T}^{2}\right)}{2 \pi} \times \\
\times \int_{x}^{1} d z\left[P_{q q}(z) \frac{x}{z} q\left(\frac{x}{z}, \mathbf{k}_{T}^{2}\right) \Theta(\Delta-z)+P_{q g}(z) \frac{x}{z} g\left(\frac{x}{z}, \mathbf{k}_{T}^{2}\right)\right], \\
f_{g}\left(x, \mathbf{k}_{T}^{2}, \mu^{2}\right)=T_{g}\left(\mathbf{k}_{T}^{2}, \mu^{2}\right) \frac{\alpha_{s}\left(\mathbf{k}_{T}^{2}\right)}{2 \pi} \times \\
\times \int_{x}^{1} d z\left[\sum_{q} P_{g q}(z) \frac{x}{z} q\left(\frac{x}{z}, \mathbf{k}_{T}^{2}\right)+P_{g g}(z) \frac{x}{z} g\left(\frac{x}{z}, \mathbf{k}_{T}^{2}\right) \Theta(\Delta-z)\right],
\end{gathered}
$$

where $P_{a b}(z)$ are the usual unregulated LO DGLAP splitting functions. The theta functions which appear in (1) and (2) imply the angular-ordering constraint $\Delta=\mu /\left(\mu+\left|\mathbf{k}_{T}\right|\right)$ specifically to the last evolution step to regulate the soft gluon singularities. For other evolution steps, the strong ordering 
in transverse momentum within the DGLAP equations automatically ensures angular ordering ${ }^{1}$. The Sudakov form factors $T_{q}\left(\mathbf{k}_{T}^{2}, \mu^{2}\right)$ and $T_{g}\left(\mathbf{k}_{T}^{2}, \mu^{2}\right)$ which appear in (1) and (2) enable us to include logarithmic loop corrections to the calculated cross sections.

The calcuation of the matrix elements generally follows the standard Feynman rules. The only difference comes from the modification of the polarization sum rules. In the $k_{T}$-factorization approach the gluon polarization density matrix takes so called BFKL form: $\sum \varepsilon^{\mu} \varepsilon^{* v}=k_{T}^{\mu} k_{T}^{v} / \mathbf{k}_{T}^{2}$. The spin density matrix for the off-shell quark with the momentum $k=x P+k_{T}$ in massless limit is [7] $\sum_{s} u^{s}(k) \bar{u}^{s}(k)=x \hat{P}$, where $P$ is the momentum of the incoming proton (or antiproton). Since the expression was obtained in the massless approximation, we neglected the light quarks masses.

In our numerical calculations we took the renormalization and factorization scales $\mu_{R}^{2}=\mu_{F}^{2}=$ $\xi^{2} p_{T}^{2}$. In order to evaluate theoretical uncertainties, we varied $\xi$ between $1 / 2$ and 2 about the default value $\xi=1$. We used the LO formula for the strong coupling constant $\alpha_{s}\left(\mu^{2}\right)$ with $n_{f}=4$ active quark flavours at $\Lambda_{Q C D}=200 \mathrm{MeV}$, so that $\alpha_{S}\left(M_{Z}\right)=0.1232$. We set the charm and beauty quark masses to $m_{c}=1.5 \mathrm{GeV}$ and $m_{b}=4.75 \mathrm{GeV}$.

In order to reduce the huge background from the secondary photons produced by the decays of $\pi^{0}$ and $\eta$ mesons the isolation criterion is introduced in the experimental analyses. This criterion is the following. A photon is isolated if the amount of hadronic transverse energy $E_{T}^{\text {had }}$ deposited inside a cone with aperture $R$ centered around the photon direction in the pseudo-rapidity and azimuthal angle plane, is smaller than some value $E_{\max }$.

$$
\begin{gathered}
E_{T}^{\text {had }} \leq E^{\max } \\
\left(\eta^{\text {had }}-\eta\right)^{2}+\left(\varphi^{\text {had }}-\varphi\right)^{2} \leq R^{2} .
\end{gathered}
$$

The isolation not only reduces the background but also significantly reduces the so called fragmentation components, connected with collinear photon radiation (10\%). We took $R=0.4$ and $E^{\max }=1 \mathrm{GeV}$.

In Figs. $1-5$ the results of our calculation [8] for the production of the prompt photon with the associated heavy quark are shown. The results are compared with the data taken by the D $\emptyset$ and CDF collaborations at $\sqrt{s}=1960 \mathrm{GeV}$ [9-12]. For comparison we also plot the NLO QCD predictions [13]. We find that the full set of experimental data is reasonably well described by the $k_{T}$-factorization approach. One can see that the shape and absolute normalization of measured cross sections are adequately reproduced. However, in the case of $\gamma+c$-jet production, the situation is a bit worse: we find a substantial disagreement between our predictions and early D $\emptyset$ data [10] at high $p^{\gamma}$ (see Fig. 4). Note, however, that very recent CDF data [11] for $\gamma+c$-jet production are well described by the $k_{T}$-factorization in a whole $p_{T}$ range (see Fig. 5). This problem remained open before recent time, but new D $\emptyset$ experimental data [14] are in a good agreement with the $k_{T}$-factorization predictions.

In Fig. 6 the results of the calculation for associated production of the prompt photon and the muon originated from the semileptonic decays of charm or beauty quarks is presented. The experimental data are from CDF [3]. To produce muons from charmed and beauty quarks, we first convert them into $D$ or $B$ hadrons using the Peterson fragmentation function [15] and then simulate

\footnotetext{
${ }^{1}$ Numerically, in (1) and (2) we applied the MSTW2008 parton distributions [6].
} 


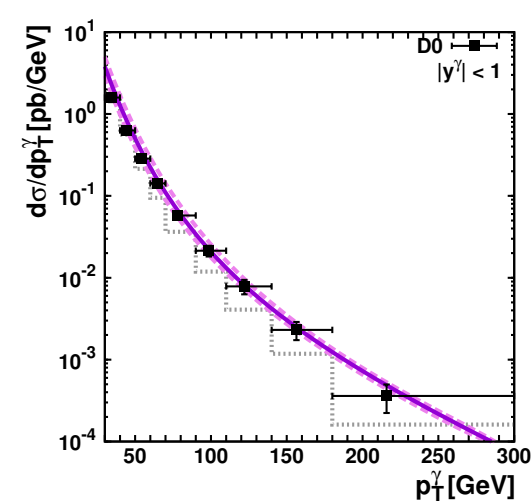

(a)

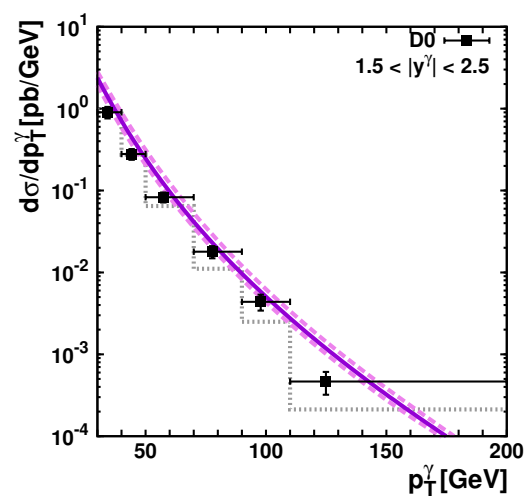

(c)

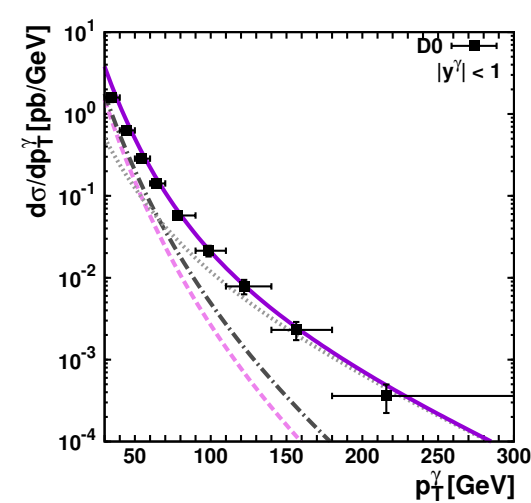

(b)

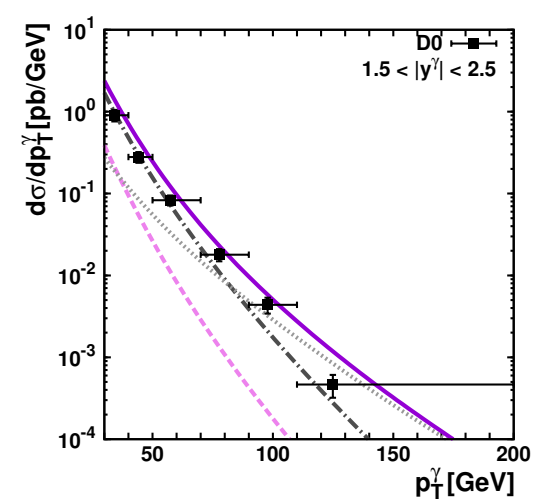

(d)

Figure 1: The associated $\gamma+b$-jet cross section as a function of photon transverse momentum $p_{T}^{\gamma}$ in the kinematical region defined by $\left|y^{\text {jet }}\right|<1.5$ and $p_{T}^{\text {jet }}>15 \mathrm{GeV}$ at $\sqrt{s}=1960 \mathrm{GeV}$. Left panels: the solid curve corresponds to the KMR predictions at the default scale $\mu=E_{T}$, whereas the upper and lower dashed curves correspond to scale variations described in the text. The dotted histogram represents the NLO pQCD predictions [13] listed in [9]. Right panels: the different contributions to the $\gamma+b$-jet cross section. The dashed, dotted and dash-dotted curves correspond to the contributions from the $g^{*} g^{*} \rightarrow \gamma Q \bar{Q}, q^{*} \bar{q}^{*} \rightarrow \gamma Q \bar{Q}$ and $q^{*} Q \rightarrow \gamma q Q$ subprocesses, respectively. The solid curve represents their sum. The experimental data are from $D \emptyset[9]$.

their semileptonic decay according to the standard electroweak theory. Additionally, the cascade decays $b \rightarrow c \rightarrow \mu$ have been taken into account. We set the fragmentation parameters $\varepsilon_{c}=0.06$ and $\varepsilon_{b}=0.006$ and corresponding branching fractions to $f(c \rightarrow \mu)=0.0969, f(b \rightarrow \mu)=0.1071$ and $f(b \rightarrow c \rightarrow \mu)=0.0802$ [16]. We find that the $k_{T}$-factorization predictions describe the data very well. One can see that the CDF data clearly favor the $k_{T}$-factorization results.

In the present work we also make some predictions for LHC energies. We define the kinematical region by the following requirements: $\left|y^{\gamma}\right|<2.5,25<p_{T}^{\gamma}<400 \mathrm{GeV},\left|y^{\text {jet }}\right|<2.2$ and $18<p_{T}^{\text {jet }}<200 \mathrm{GeV}$. Our predictions for differential $\gamma+b$-jet cross sections as a function of photon transeverse momentum $p_{T}^{\gamma}$ and rapidity $y^{\gamma}$ are shown in Fig. 7. 


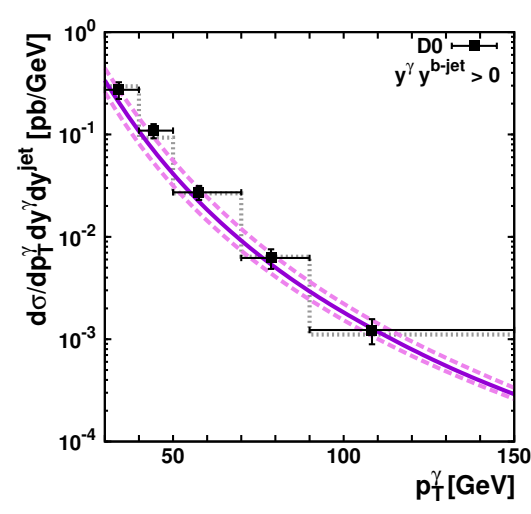

(a)

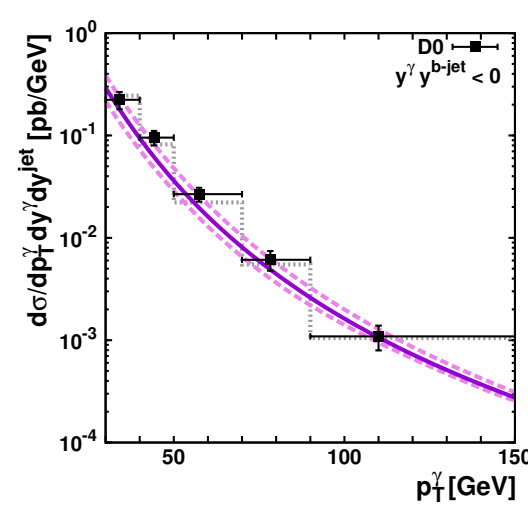

(b)

Figure 2: The associated $\gamma+b$-jet cross section as a function of photon transverse momentum $p_{T}^{\gamma}$ in the kinematical region defined by $\left|y^{\gamma}\right|<1.0,\left|y^{\text {jet }}\right|<0.8$ and $p_{T}^{\text {jet }}>15 \mathrm{GeV}$ at $\sqrt{s}=1960 \mathrm{GeV}$. The notations are the same as for the left panel of Fig. 1. The experimental data are from D [10].

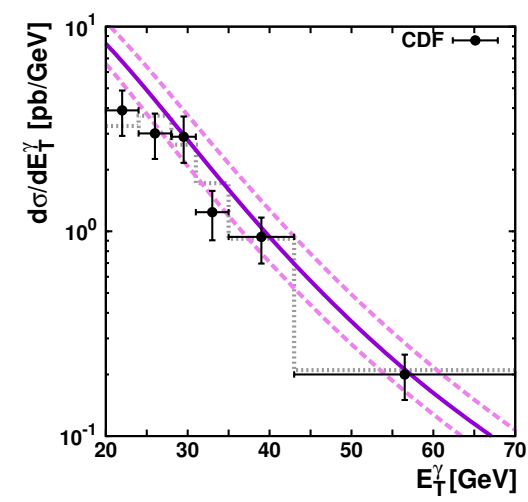

(a)

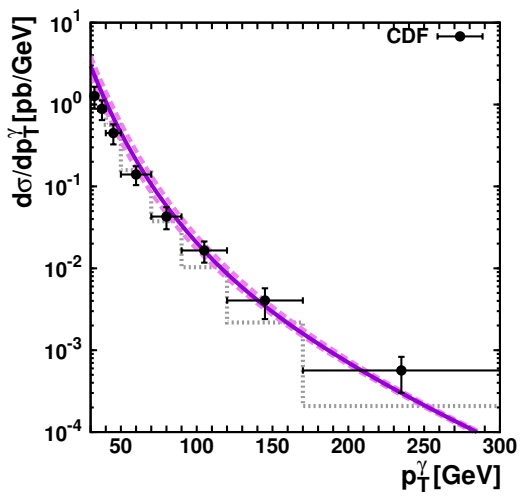

(b)

Figure 3: The associated $\gamma+b$-jet cross section as a function of photon transverse momentum $p_{T}^{\gamma}$ in the kinematical region defined by $\left|y^{\gamma}\right|<1.0,\left|y^{j e t}\right|<1.5$ and $p_{T}^{\text {jet }}>20 \mathrm{GeV}\left(\right.$ a) and $\left|\eta^{\gamma}\right|<1.1$, $\left|\eta^{\text {jet }}\right|<1.5$ and $p_{T}^{\text {jet }}>20 \mathrm{GeV}($ b) at $\sqrt{s}=1960 \mathrm{GeV}$. The notations are the same as for Fig. 2. The experimental data are from $C D F[11,12]$.

In summary, we have studied the process of the prompt photon production with the associated heavy $(b, c)$ quark in the $k_{T}$-factorization QCD approach at Tevatron and LHC energies. A reasonably good description of $\mathrm{D} \emptyset$ and $\mathrm{CDF}$ data for the associated prompt photon and heavy quark production has been obtained. Also the associated prompt photon and $\mu$-meson production has been studied. A theoretical uncertainties investigation has been studied and a predictive power of the used approach has been shown. The obtained results prove the applicability of the KMR unintegrated parton distribution for the description of such processes. 


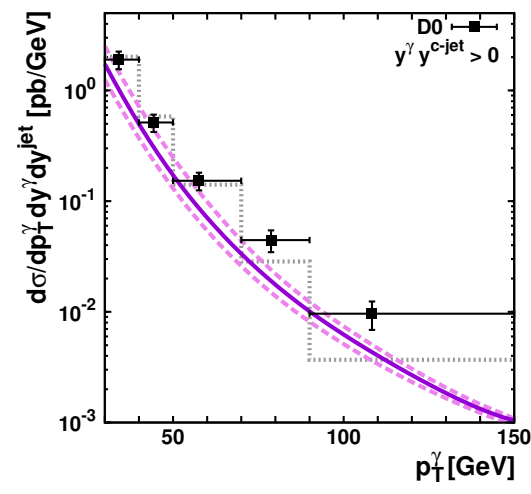

(a)

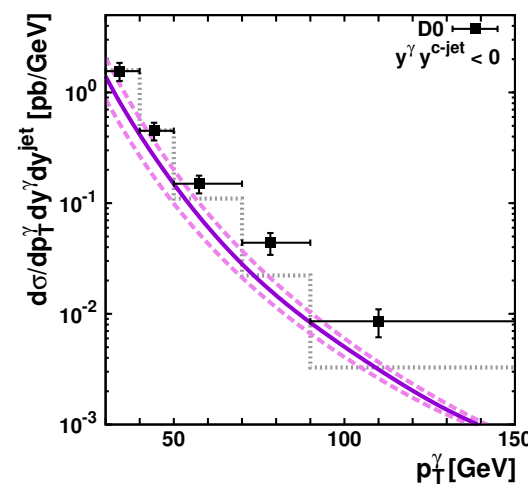

(b)

Figure 4: The associated $\gamma+c$-jet cross section as a function of photon transverse momentum $p_{T}^{\gamma}$ in the kinematical region defined by $\left|y^{\gamma}\right|<1.0,\left|y^{\text {jet }}\right|<0.8$ and $p_{T}^{\text {jet }}>15 \mathrm{GeV}$ at $\sqrt{s}=1960 \mathrm{GeV}$. The notations are the same as for Fig. 2. The experimental data are from Dり [10].

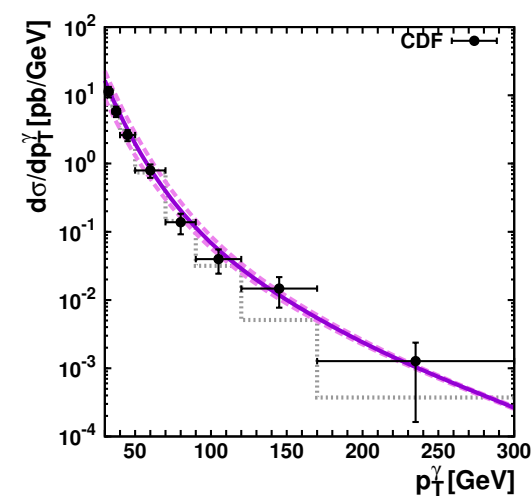

(a)

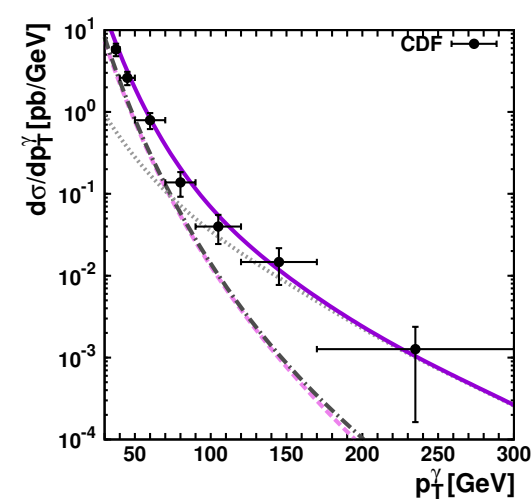

(b)

Figure 5: The associated $\gamma+c$-jet cross section as a function of photon transverse momentum $p_{T}^{\gamma}$ in the kinematical region defined by $\left|y^{\gamma}\right|<1.0,\left|y^{\text {jet }}\right|<1.5$ and $p_{T}^{\text {jet }}>20 \mathrm{GeV}$ at $\sqrt{s}=1960 \mathrm{GeV}$. The notations are the same as for Fig. 1. The experimental data are from CDF [11].

\section{Acknowledgments}

The authors would like to thank DESY Directorate for the support in the framework of Moscow - DESY project on Monte-Carlo implementation for HERA - LHC. A.V.L. and M.A.M. were supported in part by the grant of the president of the Russian Federation (MK-3977.2011.2) and RFBR grant 12-02-31030. This research was supported by the FASI of the Russian Federation (grant NS-3920.2012.2), FASI state contract 02.740.11.0244, RFBR grant 11-02-01454-a, the RMES (grant the Scientific Research on High Energy Physics) and the Ministry of education and sciences of Russia (agreement 8412). M.M. is very grateful to the Organizing Committee for the financial support. 


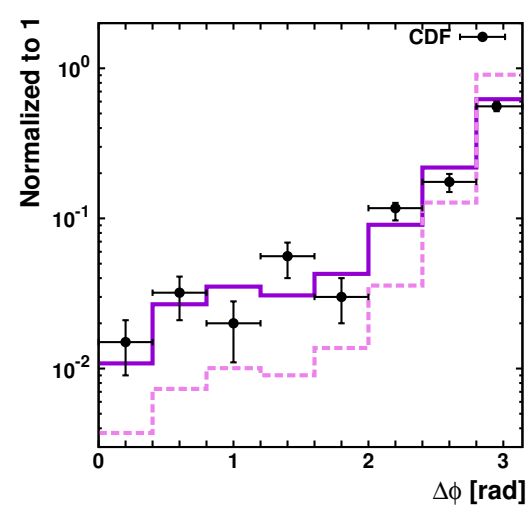

(a)

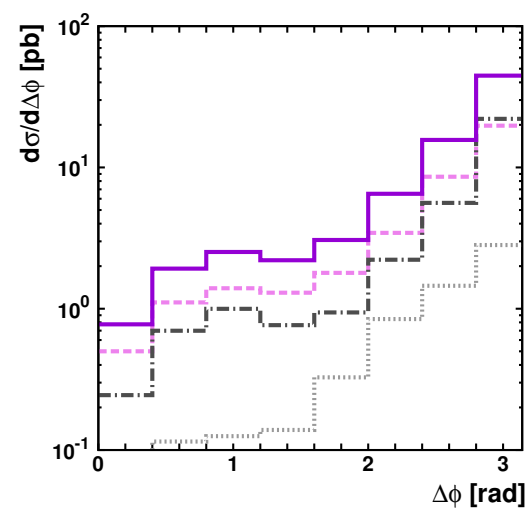

(b)

Figure 6: The associated $\gamma+\mu$ cross section as a function of the azimuthal angle difference between the produced prompt photon and muon in the kinematical region defined by $\left|\eta^{\gamma}\right|<0.9$, $\left|\eta^{\mu}\right|<1.0$ and $p_{T}^{\mu}>4 \mathrm{GeV}$ at $\sqrt{s}=1800 \mathrm{GeV}$. Panel (a): the solid line corresponds to the $k_{T}$ factorization calculation, the dashed line corresponds to the collinear QCD factorization results. The notations on the panel (b) are the same as for Fig. 1. The experimental data are from CDF [3].

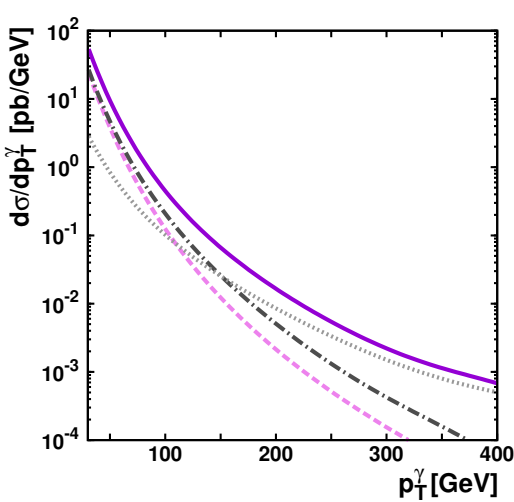

(a)

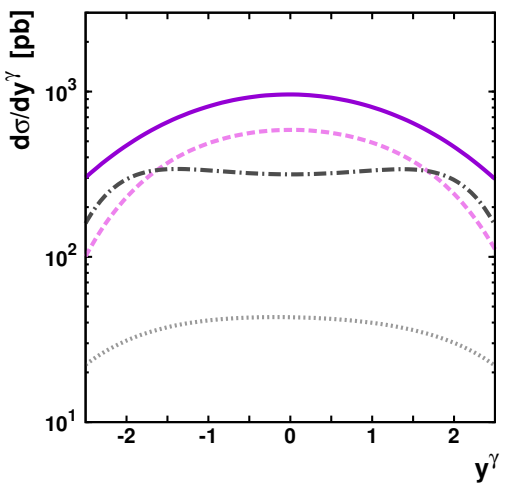

(b)

Figure 7: The associated $\gamma+b$-jet cross section as a function of photon transverse momentum $p_{T}^{\gamma}(a)$ and rapidity $y^{\gamma}(b)$ in the kinematical region defined by $\left|y^{\gamma}\right|<2.5,25<p_{T}^{\gamma}<400 \mathrm{GeV}$, $\left|y^{\text {jet }}\right|<2.2$ and $18<p_{T}^{\text {jet }}<200 \mathrm{GeV}$ at $\sqrt{s}=7000 \mathrm{GeV}$. The notations are the same as for the right panel of Fig. 1. 


\section{References}

[1] S.P. Baranov, A.V. Lipatov and N.P. Zotov, Associated production of prompt photons and heavy quarks in off-shell gluon-gluon fusion, Eur. Phys. J. C 56 (2008) 371 [0805.2650 [hep-ph ] ].

[2] T. Affolder et al. (CDF Collaboration), Cross section and heavy quark composition of $\gamma+\mu$ events

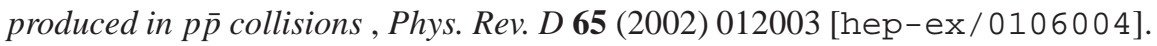

[3] F. Abe et al. (CDF Collaboration), Measurement of the associated $\gamma+\mu^{ \pm}$production cross section in $p \bar{p}$ collisions at $\sqrt{s}=1.8$ TeV, Phys. Rev. D 60 (1999) 092003 [hep-ex/9902001].

[4] M.A. Kimber, A.D. Martin and M.G. Ryskin, Unintegrated parton distributions, Phys. Rev. D 63 (2001) 114027 [hep-ph/0101348].

[5] G. Watt, A.D. Martin and M.G. Ryskin, Unintegrated parton distributions and inclusive jet production at HERA, Eur. Phys. J. C 31 (2003) 73 [hep-ph/ 0306169 ].

[6] A.D. Martin, W.J. Stirling, R.S. Thorne and G. Watt, Parton distributions for the LHC, Eur. Phys. J. C 63 (2009) 189 [0901.0002 [hep-ph]].

[7] S.P. Baranov, A.V. Lipatov and N.P. Zotov, Deep inelastic prompt photon production at HERA in the $k_{T}$-factorization approach, Phys. Rev. D 81 (2010) 094034 [1001.4782 [hep-ph] ].

[8] A.V. Lipatov, M.A. Malyshev and N.P. Zotov, Prompt photon and associated heavy quark production at hadron colliders with $k_{T}$-factorization, JHEP 1205 (2012) 104 [1204.3828 [hep-ph] ].

[9] V.M. Abazov et al. (D $\emptyset$ Collaboration), Measurement of the photon+b-jet production differential cross section in p $\bar{p}$ collisions at $\sqrt{s}=1.96$ TeV, Phys. Lett. B 714 (2012) 32 [1203.5865 [hep-ex] ].

[10] V.M. Abazov et al. (Dり Collaboration), Measurement of $\gamma+b+X$ and $\gamma+c+X$ production cross sections in p $\bar{p}$ collisions at $\sqrt{s}=1.96$ TeV, Phys. Rev. Lett. 102 (2009) $192002[0901.0739$ [hep-ex]].

[11] K. Vellidis et al. (CDF Collaboration), Photon results from CDF, in proceedings of XX International Workshop on Deep-Inelastic Scattering and Related Subjects (DIS'12).

[12] T. Aaltonen et al. (CDF Collaboration), A study of the associated production of photons and b-quark jets in p $\bar{p}$ collisions at $\sqrt{s}=1.96$ TeV, Phys. Rev. D 81 (2010) 052006 [0912.3453 [hep-ex] ].

[13] T. Stavreva and J. Owens, Direct photon production in association with a heavy quark at hadron colliders, Phys. Rev. D 79 (2009) 054017 [0901.3791 [hep-ph]].

[14] V.M. Abazov et al. (D $\emptyset$ Collaboration), Measurement of differential $\gamma+c$-jet cross sections and the ratio of $\gamma+c$ and $\gamma+b$ cross sections in $p \bar{p}$ collisions at $\sqrt{s}=1.96 \mathrm{TeV},[1210.5033$ [hep-ex]].

[15] C. Peterson, D. Schlatter, I. Schmitt and P.M. Zerwas, Scaling violations in inclusive $e^{+} e^{-}$ annihilation spectra, Phys. Rev. D 27 (1983) 105.

[16] K. Nakamura et al. (Particle Data Group Collaboration), Review of particle physics, J. Phys. G 37 (2010) 075021. 